\title{
The predictive value of hemodynamics change on fluid responsiveness during renal replacement therapy including both blood drainage and reinfusion in critically ill patients
}

\author{
Daozheng Huang ${ }^{1 * *}$, Huan $\mathrm{Ma}^{2 *}$, Liyan Hong ${ }^{3}$, Yan Wu${ }^{1}$, Shouhong Wang ${ }^{1}$ and Tiehe Qin ${ }^{1}$ \\ ${ }^{1}$ Department of Critical Care Medicine, Guangdong Geriatric Institute, Guangdong General Hospital, Guangdong Academy of Medical Sciences, Guangzhou, China \\ ${ }^{2}$ Department of Cardiology, Guangdong Cardiovascular Institute, Guangdong General Hospital, Guangdong Academy of Medical Sciences, Guangzhou, China \\ 3Department of Critical Care Medicine, Changjiang County People's Hospital, Changjiang, China \\ \#These authors contributed equally to this work.
}

This ongoing clinical trial entitled "The predictive value of hemodynamics change on fluid reactivity during blood purification including both blood drainage and reinfusion in severe patients (Chinese Clinical Trial Registration Number: ChiCTR-DDD-17010534)" will evaluate the value of inverse-passive leg raising (IPLR) test and likepassive leg raising (LPLR) test in predicting fluid responsiveness during blood reinfusion maneuver performed after renal replacement therapy (RRT) or blood drainage before RRT in patients with septic shock, which may provide a new way of thinking and method for the evaluation of fluid responsiveness in critically ill patients.

Stroke volume variation (SVV) appears to be a good predictor of fluid responsiveness in critically ill patients [1]. However, SVV is often not valuable in predicting fluid responsiveness in some settings, such as spontaneous breathing, arrhythmia, etc. At present, there are only two techniques which are widely used, practical, easy to perform, and physiologically based, which can be used to predict critically ill patients' fluid responsiveness with a high degree of accuracy, namely, the PLR maneuver and the fluid challenge (FC) $[2,3]$. Both PLR and FC are based on the cardiac function curve of Frank-Starling. However, fluid administration does not always lead to increase cardiac output (CO). This depends on the curvilinearity of the Frank-Starling relationship: If the level of cardiac function of patient is on the initial and steep part of the Frank-Starling curve, increasing preload through FC or PLR test can cause corresponding increase in stroke volume (SV) or CO. On the contrary, if the heart is operating on the distal and flat part of the curve, no significant increase in SV is expected from fluid administration or PLR test, or even there are adverse effects [4]. It is dangerous and even against the ethical medical principle for patients with circulatory failure to allow FC to increase the preload within a short time in order to evaluate the fluid responsiveness. PLR can solve the above limitations and allow reliable prediction of fluid responsiveness even in patients with spontaneous breathing activity or arrhythmias [5]. However, clinically PLR test may not be implemented successfully for all critically ill patients, such as patients with intracranial hypertension, brake patients and parts of surgery patients. And the PLR test cannot accurately predict fluid responsiveness in patients with intra-abdominal hypertension [6]. On the other hand, if the preload can be reduced within a short time, one can evaluate the fluid responsiveness by judging the curvilinearity of the Frank-Starling relationship for the patients through SV or CO parameter changes. For RRT patients, the preload is reduced within a short time during blood drainage before the treatment. In theory, this approach might be able to predict fluid responsiveness. The process of blood reinfusion after RRT simulates the effect of autologous blood transfusion, without changing patients' positions. Hence, we can infer that its value in evaluating fluid responsiveness is similar to effect of PLR test.

Therefore, it is of significance to investigate that if one can evaluate the fluid responsiveness accurately based on the decreasing or increasing effect of preload during process of blood drainage or reinfusion in RRT.

The IPLR and LPLR tests are diagnostic tests, which evaluate the value of IPLR and LPLR in predicting the fluid responsiveness in patients with septic shock. Regardless of the outcome, it may pose an impact on the diagnosing method to evaluate fluid responsiveness, because this method is based on the Frank-Starling law of heart, but use reverse thinking to discuss fluid responsiveness, which is a beneficial complement to the traditional methodology to evaluate fluid responsiveness. If the results are satisfying, IPLR or LPLR test can be considered to be used to evaluate fluid responsiveness for patients with PLR contraindications but in need of RRT, or patients in RRT with septic shock. This may provide a new method to predict fluid responsiveness.

\section{Funding}

This work was supported by the grant from Medical Scientific Research Foundation of Guangdong Province, People's Republic of China (Grant number: A2018064), the National Natural Science Foundation of China (Grant number: 81602848) and the grant from Guangdong Provincial Bureau of Traditional Chinese Medicine, People's Republic of China (Grant number: 20172072 ).

${ }^{\star}$ Correspondence to: Tiehe Qin, Department of Critical Care Medicine, Guangdong Geriatric Institute, Guangdong General Hospital, Guangdong Academy of Medical Sciences, Guangzhou 510080, China. E-mail: dr.qin@qq.com

Received: September 16, 2018; Accepted: September 25, 2018; Published: September 28, 2018 
Huang D (2018) The predictive value of hemodynamics change on fluid responsiveness during renal replacement therapy including both blood drainage and reinfusion in critically ill patients

\section{Competing interests}

The authors declare that they have no competing interests.

\section{References}

1. Zhang Z, Lu B, Sheng X, Jin N (2011) Accuracy of stroke volume variation in predicting fluid responsiveness: a systematic review and meta-analysis. $J$ Anesth 25 : 904-16. [Crossref]

2. Marik PE, Monnet X, Teboul JL (2011) Hemodynamic parameters to guide fluid therapy. Ann Intensive Care 1: 1. [Crossref]
3. Monnet X, Teboul JL (2015) Passive leg raising: five rules, not a drop of fluid! Crit Care 19: 18 [Crossref]

4. Marik PE (2016) Fluid Responsiveness and the Six Guiding Principles of Fluid Resuscitation. Crit Care Med 44: 1920-1922. [Crossref]

5. Monnet, Xavier, Teboul, Jean-Louis (2008) Passive leg raising. Intensive Care Med 34 659-663. [Crossref]

6. Yazine M, Airapetian T, Airapetian N, Lorne E, Hijazi M, et al. (2010) The passive leg-raising maneuver cannot accurately predict fluid responsiveness in patients with intra-abdominal hypertension. Crit Care Med 38: 1824-1829. [Crossref]

Copyright: @2018 Huang D. This is an open-access article distributed under the terms of the Creative Commons Attribution License, which permits unrestricted use, distribution, and reproduction in any medium, provided the original author and source are credited. 